\title{
Vertical interface augmented tunability of scattering spectra in ferromagnetic microwire/silicone rubber metacomposites
}

\author{
Azim Uddin, Faxiang Qin ${ }^{*}$, Diana Estevez, and Hua-Xin Peng \\ Institute for Composites Science Innovation (InCSI), School of Materials Science and Engineering, Zhejiang University, \\ 38 Zheda Road, Hangzhou 310027, PR China
}

Received: 10 November 2020 / Accepted: 16 December 2020

\begin{abstract}
Previously, we have demonstrated a viable approach based on microstructural and topological modulation of periodically arranged elements to program wave scattering in ferromagnetic glass-coated microwire metacomposites. In order to fully exploit the intrinsic structure of the composite, here, we implement the concept of composites plainification by an in-built vertical interface on randomly dispersed short-cut microwires allowing the adjustment of electromagnetic properties to a larger extent. Such interface was modified through arranging wires with different internal structures in two separated regions and by alternating these regions through wire concentration variations associated with polarization differences across the interface. When the wire concentration was equal in both regions, two well-defined transmission windows with varied amplitude and bandwidth were generated. Wire concentration fluctuations resulted in strong scattering changes ranging from broad passbands to pronounced stopbands, demonstrating the intimate relationship between wire content and space charge variations at the interface. This provides a new method to rationally exploit interfacial effects and microstructural features of microwire metacomposites. Moreover, the advantages of enabling tunable scattering spectra by merely $0.053 \mathrm{vol} . \%$ of fillers and simple structure make the proposed plainification strategy instrumental to designing filters with broadband frequency selectivity.
\end{abstract}

Keywords: Ferromagnetic microwire metacomposites / Electromagnetic wave scattering / Band-stop filters / Band-pass filters / Interfacial polarization

\section{Introduction}

Metacomposites containing ferromagnetic glass-coated microwires have attracted much attention owing to their multiple emerging properties and associated broad range of applications such as structural health monitoring, microwave absorption and electromagnetic shielding [1-5]. Strong responses from the microwires to the incident electromagnetic wave are possible due to their distinguished giant magneto- and stress-impedance effects, soft magnetic character and emerging double-negative (DNG) properties arising from their arrangement in the matrix [4,6-8]. Moreover, it is possible to further tune the scattering spectra of microwire metacomposites through external stimuli such as magnetic field, temperature or stress. However, such approaches entail practical burdens, limiting their applications in compact and light-weight devices.

\footnotetext{
is Supporting information is available in electronic form at https://www.epjam.edp-open.org/10.1051/epjam/2021003

* Corresponding author: Faxiangqin@zju.edu.cn
}

Beyond those methods, we have recently proposed a programming-based strategy using the microwire (microstructurally modified by current annealing) as a code unit and different wire combinations as the code pattern $[9,10]$. Although such approach enabled the modulation of scattering properties to a large extent, strategies other than increasing the number of components and modifying microstructure have not yet been exploited. In this context, we have also demonstrated the novelty of designing vertical interfaces on carbon nanocomposites to trigger significant alteration of microwave dielectric properties and hence scattering properties [11]. Space charges accumulate near the interface and by restraining their local distribution distinct dielectric responses can be obtained. This kind of so-called "plainified" composites design makes full use of the composite structure for maximizing specific performance while maintaining the same filler content. Most recently, such concept was implemented for effective structural adjustment of porous RGO aerogels to tune the complex permittivity of the composites in developing highly efficient electromagnetic wave absorbents [12,13]. Metal alloys have also taken advantage of such approach by altering grain boundaries with fewer or even zero alloying elements $[14,15]$. 
Randomly dispersed metacomposites, the so-called intrinsic/random metamaterials with DNG properties where functional fillers are randomly distributed in an insulating matrix, have been investigated by Xie et al. $[16,17]$. In such metamaterials, the filler's size is no longer dependent on wavelength because the negative electromagnetic parameter directly originates from the intrinsic property of component materials [18]. As a result, random metacomposites have agitated enormous interests in recent years as a novel and flexible approach for adjusting electromagnetic parameters $[17,19]$. However, the random dispersion of functional fillers means that it cannot be precisely controlled. Moreover, the scattering mechanism in metacomposites incorporating randomly arranged microwires remains unclear.

In this work, we move forward to extrapolate the plainification design on microwire-based metacomposites and the effect of randomness on their scattering properties. Initially, by using the same type of microwires and modifying its microstructure through current annealing, additional filler types are avoided. Secondly, by randomly arranging the as-cast and annealed wires in two regions of the composite, an extra factor influencing the wave propagation comes into play, i.e., interface-induced polarization. This polarization originates from the differences in conductivity, permittivity and relaxation time of charge carriers across the in-built vertical interface [11]. We then designed several representative vertical-interface composite systems by enlarging or reducing one of the regions through variations in the corresponding wire volume concentration. Scattering spectra having bandstop and band-pass regions with frequency and bandwidth tuning flexibility could be achieved through wire loadings as low as 0.053 vol. \% . Therefore, a design method based on single-wire modification and the concept of plainification by in-built interfaces is sufficient to provide a broad scope of electromagnetic signatures. This could have substantial implications for the design of efficient and low-cost bandstop/band-pass filters. Moreover, the frequency shift could be conveniently used in practical applications such as stress monitoring, whereby the transformation of the scattering spectra will be similar but due to the tensile stress. These findings offer new opportunities of materials plainification design for developing large-scale metacomposites without compromising lightweight feature or structural integrity.

\section{Experimental method}

\subsection{Fabrication and current annealing of $\mathrm{Co}_{60} \mathrm{Fe}_{15} \mathrm{Si}_{10} \mathrm{~B}_{15}$ glass-coated microwires}

$\mathrm{Co}_{60} \mathrm{Fe}_{15} \mathrm{Si}_{10} \mathrm{~B}_{15}$ glass-coated microwires with a total wire diameter of $35.4 \mu \mathrm{m}$ and metallic core diameter of $27.2 \mu \mathrm{m}$ were fabricated using a modified Taylor-Ulitovskiy technique [20]. Such fabrication method allows the production of continuous wires with easily controllable dimensions [20,21]. The produced microwires were currentannealed under different DC currents of 30 and $40 \mathrm{~mA}$ for 10 min by mechanically removing the glass coat from the wire ends to allow electrical contact. The changes in DC resistance of the current-annealed microwires were monitored with a digital multi-meter showing a considerable drop for the $40 \mathrm{~mA}$ treated-wire which is related to the onset of crystallization and larger grain size due to the rise in temperature $[9,22]$. The microstructure of the as-cast and current-annealed wires consists mainly of nanocrystalline droplets embedded in amorphous matrix [23]. Magnetic properties of the microwires were evaluated by Quantum Design PPMS-VSM at room temperature.

\subsection{Preparation and electromagnetic characterization of randomly dispersed short-cut $\mathrm{Co}_{60} \mathrm{Fe}_{15} \mathrm{Si}_{10} \mathrm{~B}_{15}$ glass-coated microwires/silicone rubber composites}

Short-cut microwires of about $5 \mathrm{~mm}$ length were randomly dispersed in the matrix. SYLGARD(R) 184 silicone elastomer kit (Dow Corning) was used as the matrix containing base and curing agent which were thoroughly mixed using a weight ratio of 10:1. For composites with a single type of wires, the silicone was poured into a designed cuboid mold and cured partially before the placement of the wires to avoid them from sinking to the bottom. For the composites containing separate regions with different type of microwires, the cuboid mold was split vertically by a separator placed at a preset distance. The silicone was then poured into the mold and cured partially and the separator was subsequently removed once the wires were poured in the resin. Composite samples with dimensions of $22.86 \times 10.16 \times 2 \mathrm{~mm}^{3}$ were fabricated after curing at $125^{\circ} \mathrm{C}$ for $20 \mathrm{~min}$. Two kinds of composites were prepared. The first type incorporating 50 wires ( $0.053 \mathrm{vol} . \%)$ of the same type, i.e., as-cast A, $30 \mathrm{~mA}$-current annealed B or $40 \mathrm{~mA}$-current annealed C (Fig. 1a). The inset shows the selected-area electron diffraction (SAED) pattern of as-cast (A) and the high-resolution transmission electron microscopy (HRTEM) images of the $30 \mathrm{~mA}$ (B) and $40 \mathrm{~mA}$ (C)-annealed wires respectively. For the as-cast wires, nanocrystalline spots are embedded in the predominant amorphous phase (pointed by red arrows). For the $40 \mathrm{~mA}$ annealed sample, very fine particles smaller than $5 \mathrm{~nm}$ are uniformly distributed within the amorphous matrix. The darker droplet-like nanoparticles in the annealed samples mainly consist of heavier elements i.e., Co and Fe, while the brighter droplets correspond to the lighter elements Si and B $[9,24]$. The second type of composite corresponds to incorporating the as-cast $\mathrm{A}$ and annealed short-cut wires $\mathrm{X}$ (where $\mathrm{X}$ corresponds to $\mathrm{B}$ or $\mathrm{C}$ annealed wires) in separated regions and variable proportion in the matrix (Fig. 1b and Tab. 1).

Figure 2 shows the optical microscope images for the composites incorporating the randomly dispersed short-cut microwire arrays. Figure 2a shows the composites containing a single type of short-cut wires while Figure $2 \mathrm{~b}-\mathrm{d}$ shows the composites including as-cast (A) and current-annealed wires $(\mathrm{X})$ in different regions forming a vertical interface in which polarization takes place. The images display the uniform distribution of the microwires in the polymer matrix. 
(a)

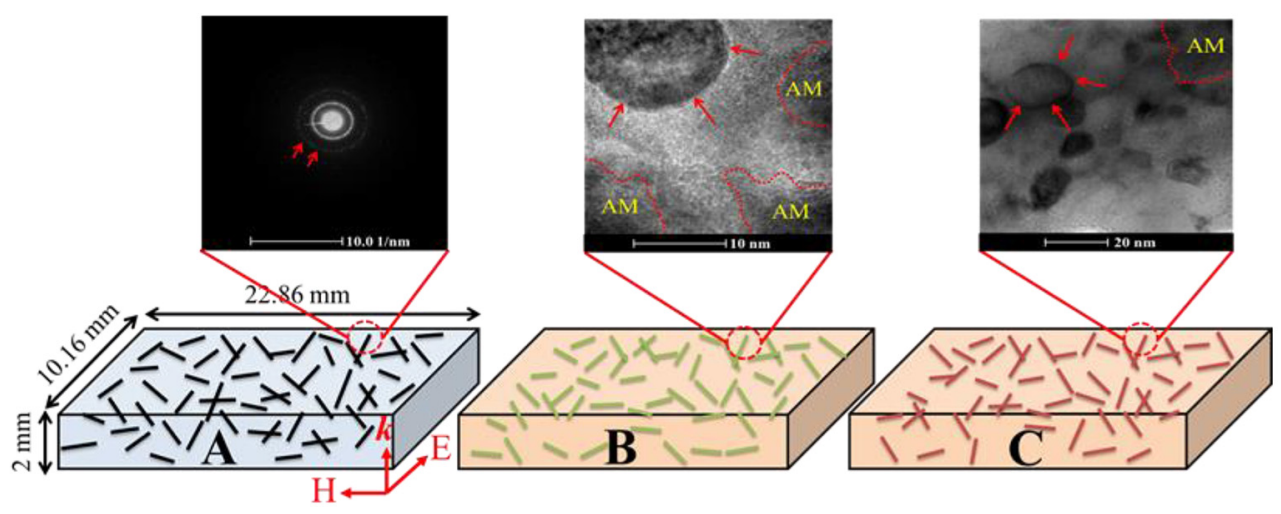

(b)

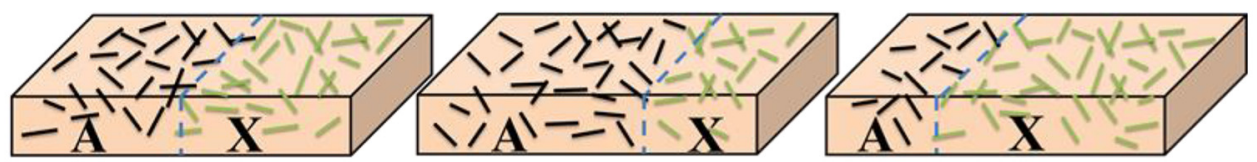

Fig. 1. Randomly dispersed short-cut microwire composites. (a) Composites incorporating the same type of wire, as-cast A, 30 mAannealed B or $40 \mathrm{~mA}$-annealed $\mathrm{C}$ with a total wire amount of 0.053 vol. \%. The insets are the SAED pattern for the as-cast wire A, HRTEM images of 30mA-annealed wire B and $40 \mathrm{~mA}$-annealed wire C, where "AM" corresponds to the amorphous phases; (b) Composites combining the as-cast $\mathrm{A}$ and annealed wires $\mathrm{X}$ (where $\mathrm{X}$ corresponds to $\mathrm{B}$ or $\mathrm{C}$ annealed wires) in separated regions and different proportions. The dotted line represents the vertical interface formed between the two different regions.

Table 1. Filler proportion in the composites combining as-cast A and annealed short-cut wires X (B: $30 \mathrm{~mA}$ or C: $40 \mathrm{~mA}$ ) distributed in separated regions.

\begin{tabular}{llll}
\hline As-cast Wire-A amount (vol. \%) & \multicolumn{2}{c}{ Annealed Wires-X amount (vol. \%) } & Total amount (vol. \%) \\
\cline { 2 - 3 } & Wire-B & Wire-C & \\
\hline $25(0.026)$ & $25(0.026)$ & - & $50(0.053)$ \\
$25(0.026)$ & - & $25(0.026)$ & $50(0.053)$ \\
$33(0.035)$ & $17(0.018)$ & - & $50(0.053)$ \\
$33(0.035)$ & - & $17(0.018)$ & $50(0.053)$ \\
$17(0.018)$ & $33(0.035)$ & - & $50(0.053)$ \\
$17(0.018)$ & - & $33(0.035)$ & $50(0.053)$ \\
\hline
\end{tabular}

Scattering S-parameters of the composites were measured with Rohde \& Schwarz ZNB 40 vector network analyzer (VNA) by using a WR-90 waveguide in $\mathrm{TE}_{10}$ dominant mode from 8.2 to $12.4 \mathrm{GHz}$ (Fig. 3). Before the measurements, the VNA was calibrated by the TRL (thrureflect-line) calibration method [25].

\section{Results \& discussions}

\subsection{Magnetic and electrical properties of as-cast and current annealed $\mathrm{Co}_{60} \mathrm{Fe}_{15} \mathrm{Si}_{10} \mathrm{~B}_{15}$ glass-coated microwires}

Figure 4 shows the magnetic hysteresis loops, magnetoimpedance and electrical conductivity for the as-cast and current annealed microwires. Current annealing improves the magnetic softness evidenced by the drastic decrease in coercivity from 15 Oe for the as-cast wire to 0.8 Oe for the $40 \mathrm{~mA}$-annealed wire and by the drop in magnetic anisotropy (Fig. 4a). Annealing relieves the internal stresses originated during fabrication and modifies the original domain structure of the glass-coated microwires, resulting in the change of the magnetic softness as shown in (Fig. 4a). The annealing tends to decrease magnetoelastic coupling and anisotropy field, improving the magnetic softness of the wires. Generally, the magnetic behavior of microwires depends intimately on the domain structure, which is determined by minimization of the magnetoelastic energy $K_{m e}$ given by:

$$
K_{m e}=\frac{3}{2} \lambda_{s} \sigma_{i i}
$$

where $\lambda_{s}$ and $\sigma_{i i}$ refer to the magnetostriction coefficient and the dominant internal stress component, respectively $[9,26]$. The internal stresses $\sigma_{i i}$ of the wires can be redistributed or relieved by modifying the microstructure of the wires through annealing improving the magnetic softness. 

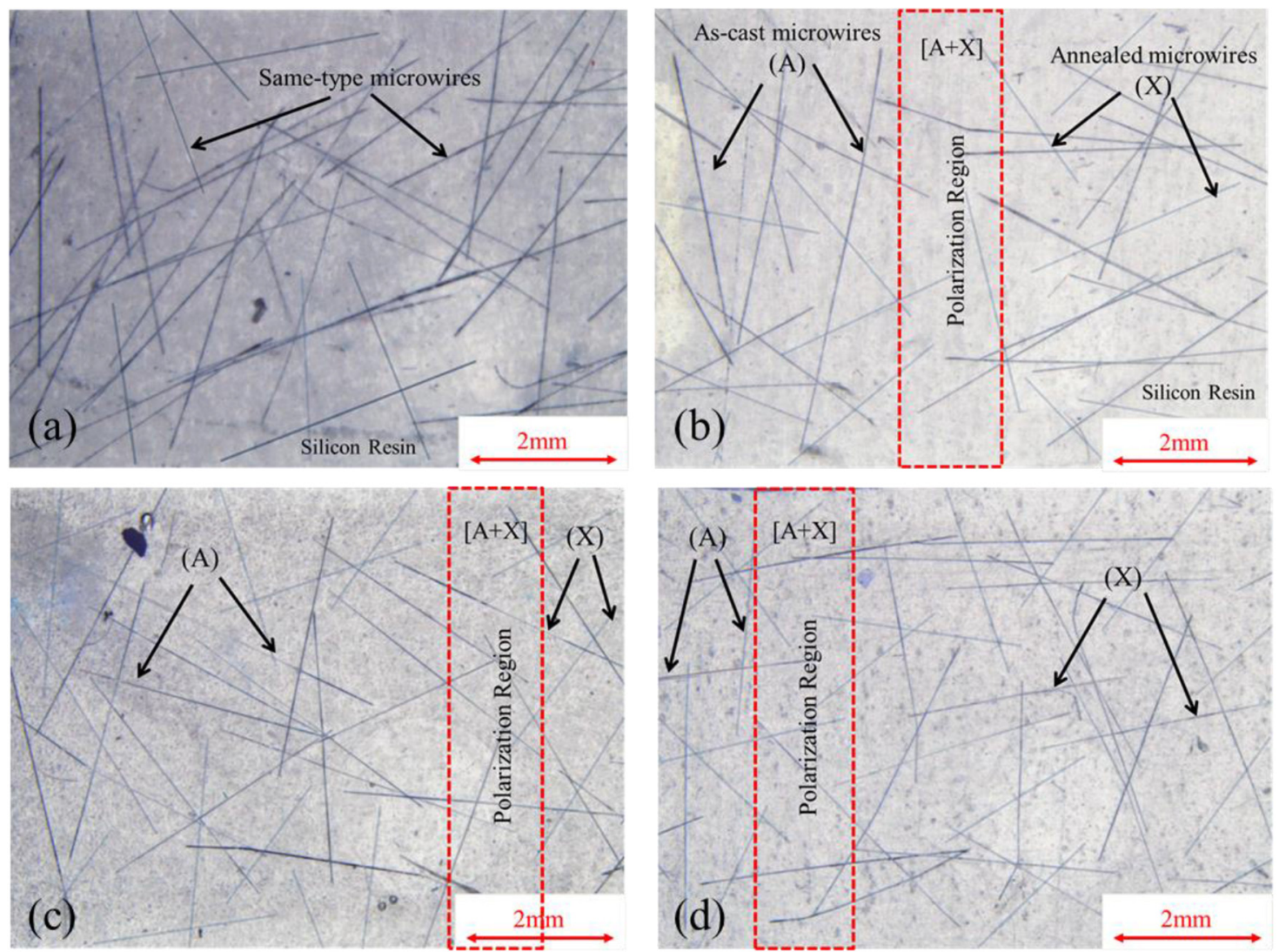

Fig. 2. Optical images of composites containing randomly distributed (a) same-type wires and (b-d) the incorporation of as-cast wires A and annealed wires X (where X corresponds to B or C annealed wires) in separate regions and different proportions. The dotted outline represents the vertical interfaces formed between the two different regions.

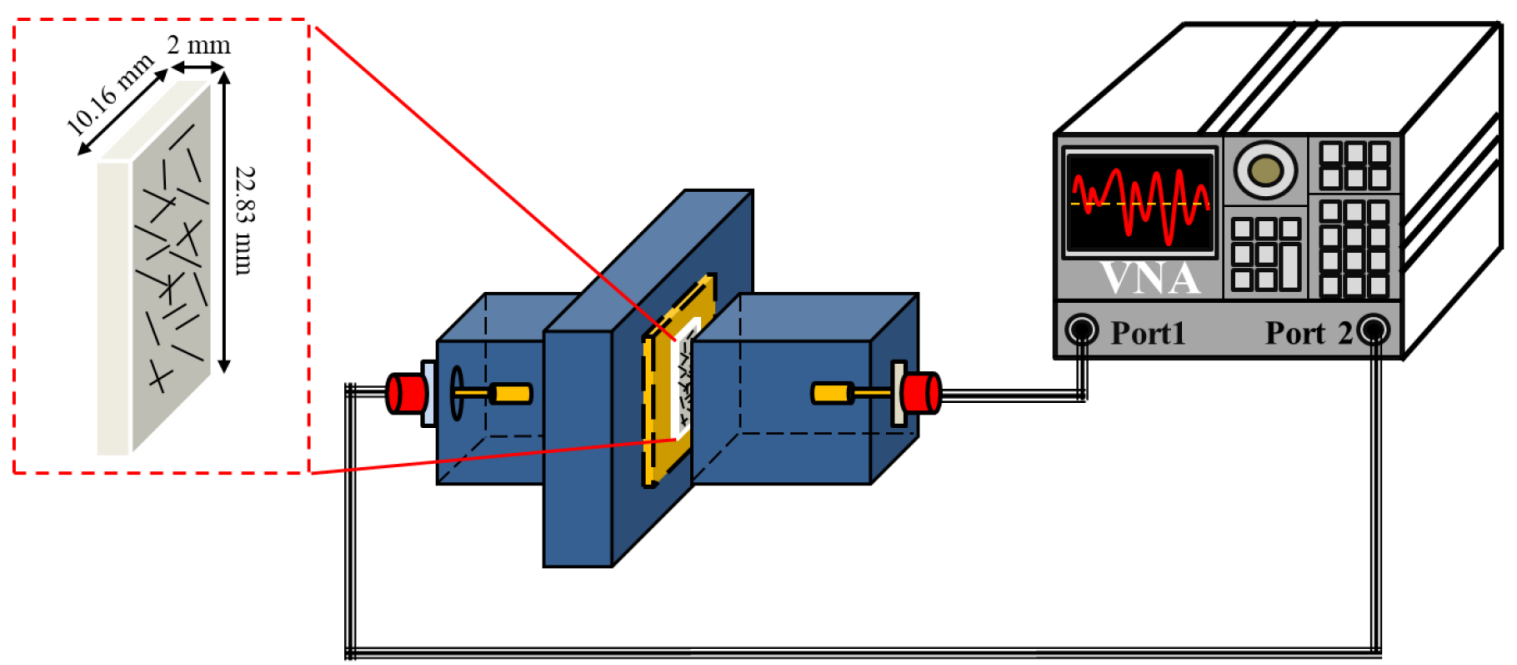

Fig. 3. Measurement setup for obtaining the scattering parameters of the microwire composite sample. The waveguide system includes two coaxial adapters (Port 1 and 2) connected to a vector network analyzer (frequency range: $8.2-12.4 \mathrm{GHz}$ ). The size of the sample measured in the waveguide was $22.86 \times 10.16 \times 2 \mathrm{~mm}^{3}$. 

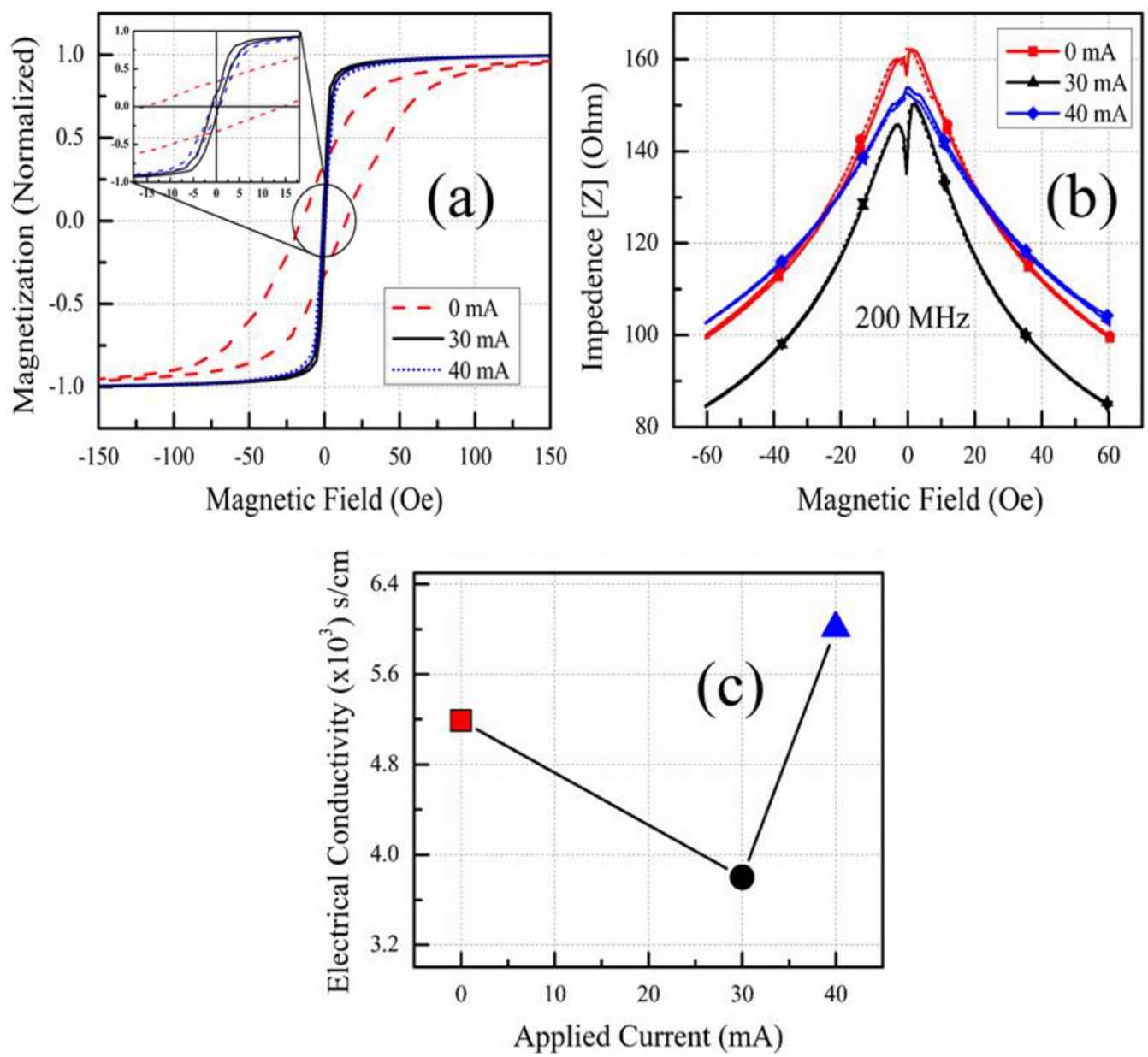

Fig. 4. (a) Magnetic hysteresis M-H loops, (b) magneto-impedance at $200 \mathrm{MHz}$ and (c) electrical properties of as-cast, 30 and 40 current annealed $\mathrm{Co}_{60} \mathrm{Fe}_{15} \mathrm{Si}_{10} \mathrm{~B}_{15}$ microwires. Inset in Figure 4a shows the zoomed-in M-H loops at low field.

From the two branches of the magneto-impedance curves (under increasing and decreasing magnetic fields) at $200 \mathrm{MHz}$ (Fig. 4b), the as-cast wire is characterized by two peaks of weak intensity, which indicates a system with a weak circumferential magnetic anisotropy [26,27]. Current annealing the wire at $30 \mathrm{~mA}$ results in pronounced asymmetrical double peaks and lower impedance, demonstrating the contribution to the magnetization of the circular domains at the wire surface shell $[26,28,29]$.

Further annealing at $40 \mathrm{~mA}$ seems to deteriorate the magnetization along the circumference likely due to the above-mentioned onset of crystallization in that sample. Such changes in magnetization and impedance curves are related to relief of internal stresses and structural relaxation [30,31]. Changes in electrical conductivity also reflect the structural modification of the microwires during current annealing (Fig. 4c). The increase in conductivity in the $40 \mathrm{~mA}$-annealed wire originates from the larger grain size, which is caused by Joule heating leading to less electron scattering at the reduced grain boundaries [9,32]. The structural changes due to annealing as indicated in both magnetic and electrical properties of the microwires will have a considerable influence on the electromagnetic properties of their composites, as will be discussed in the following section.

\subsection{Scattering properties of randomly dispersed short-cut $\mathrm{Co}_{60} \mathrm{Fe}_{15} \mathrm{Si}_{10} \mathrm{~B}_{15}$ glass-coated microwires/silicone rubber composites}

The parameters that describe the material properties in microwaves frequencies are found from the measurement of the transmission $\mathrm{S}_{21}$ and reflection $\mathrm{S}_{11}$ coefficients. Figure 5 presents the scattering properties of the composites containing randomly dispersed microwires of the same type and a combination of as-cast and annealed wires. For the same type-wire composites, a passband extending from 8.5 to $11 \mathrm{GHz}$ frequency range appears in the transmission spectrum of composites containing as-cast wires A (Fig. 5a). From Figure 2, the wires are well-dispersed in the polymer matrix which is reflected by similar trends and reproducible EM properties when measuring several samples for each series (Fig. S1). This band-pass filter allows such specific band of frequencies to pass through 


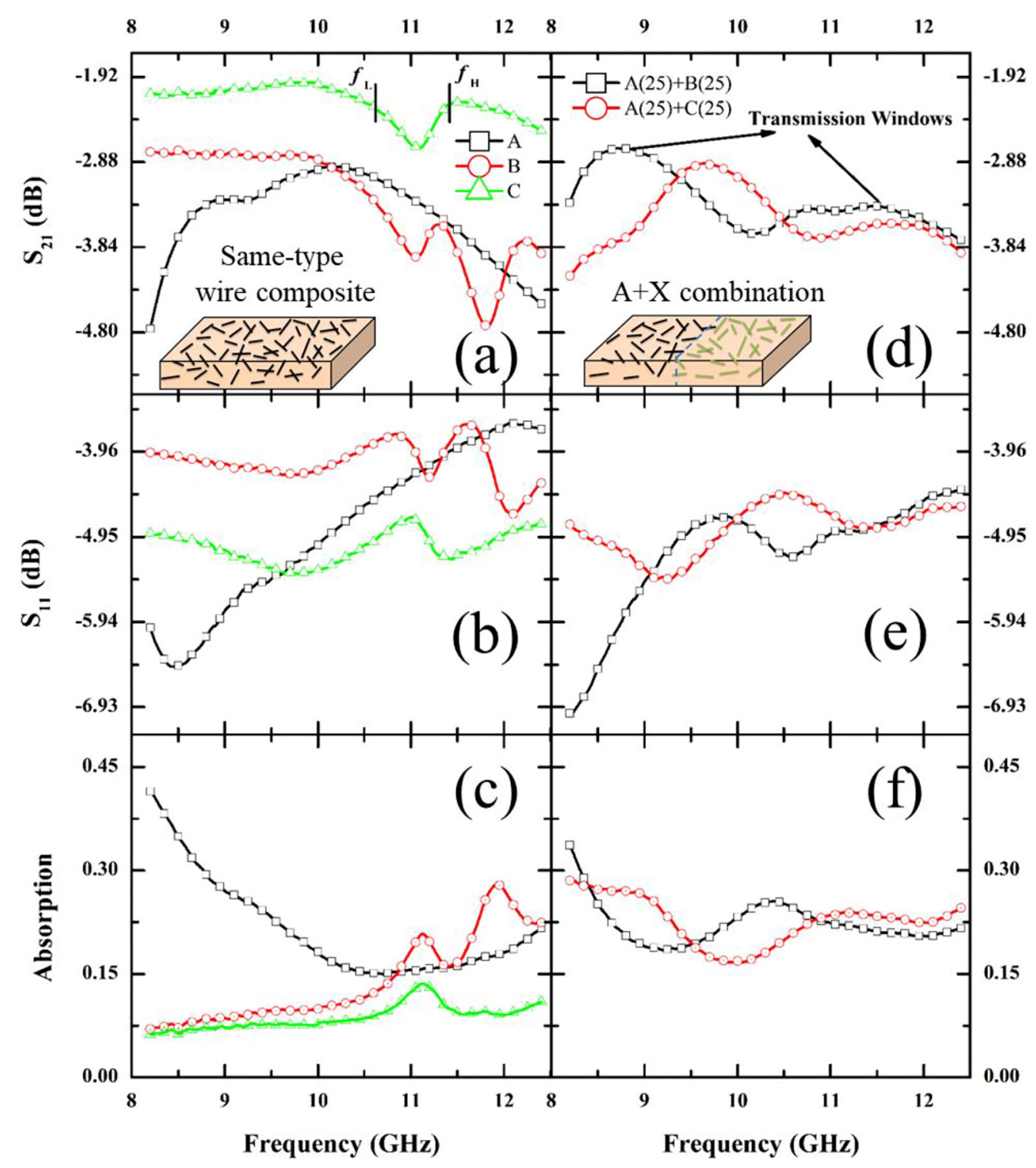

Fig. 5. Scattering properties: transmission $S_{21}$, reflection $S_{11}$, and absorption of the composites containing only one type of wires (left panel) and an equal combination of as-cast A and annealed X (right panel) randomly dispersed short-cut microwires. The labels $f_{\mathrm{L}}$ and $f_{\mathrm{H}}$ correspond to the two cut-off, frequency points of the stopband feature.

while rejects the rest. When the inclusions are replaced by the annealed microwires, a switching from passband to stopband feature with transmission dip or resonance and associated reflection and absorption peaks is identified. Band-stop filters block frequencies that lie between its two cut-off frequency points $\left(f_{\mathrm{L}}\right.$ and $\left.f_{\mathrm{H}}\right)$ but pass all those frequencies either side of this range. At the resonance, the current distribution in wires strongly depends on their surface impedance and, in general, the lower the surface impedance, the greater the scattering properties [28]. In microwires, the surface impedance reaches a minimum when the magnetization is along the circular direction, which corresponds to a circumferential anisotropy. This is confirmed by hysteresis loops of the annealed microwires (Fig. 4a) presenting almost linear shape with low coercivity and anisotropy field as a result of Joule heating. Thus, a deep minimum in transmission is seen for the composites containing the annealed short-cut microwires.

Moreover, short pieces of microwires interact with the electromagnetic radiation acting as micro-antennas. Thus, the wire length $l$ and permittivity of the dielectric matrix $\varepsilon_{m}$ define the characteristic frequency related to the antenna resonance:

$$
f_{\text {res }}=c /\left(2 l \sqrt{\varepsilon_{m}}\right),
$$

where $c$ corresponds to the speed of light [7]. For $l=5 \mathrm{~mm}$ and $\varepsilon_{m}=2$, the resonance frequency is close to $20 \mathrm{GHz}$, which falls out of the measurement range of the present work. The discrepancy between the theoretical value of the 


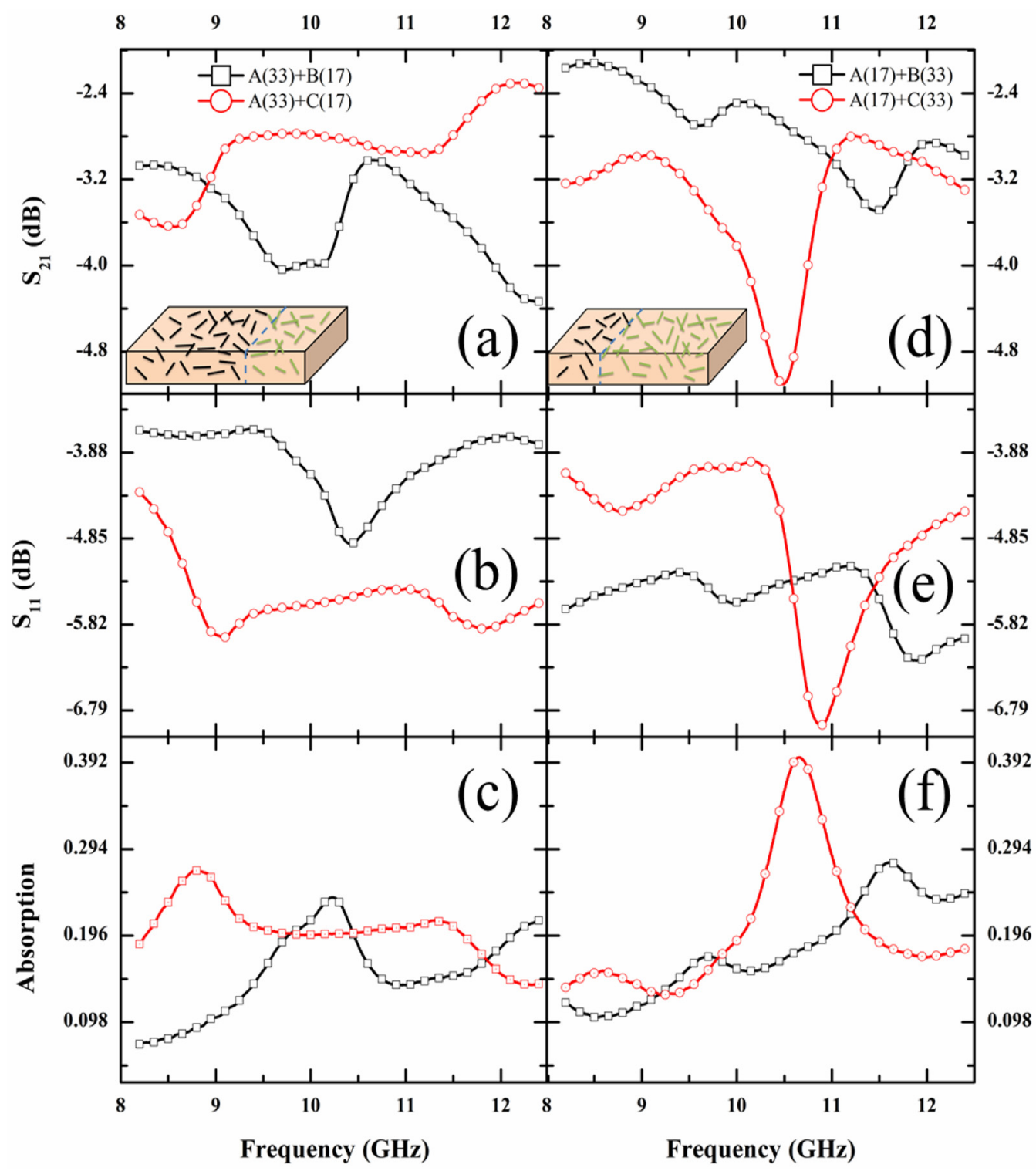

Fig. 6. Scattering properties: transmission $S_{21}$, reflection $S_{11}$ and absorption of the composites containing different combinations of as-cast $\mathrm{A}$ and annealed X $(30 \mathrm{~mA}, \mathrm{~B}$ or $40 \mathrm{~mA}, \mathrm{C})$ randomly dispersed short-cut microwires in separated regions and different proportions, i.e., 33 pieces of as-cast and 17 pieces of annealed wires (right panel) and 17 as-cast and 33 annealed wires (left panel).

resonance and the one measured for the random-wire composites can be attributed to the fact that the long-range dipolar-dipolar interaction gives rise to additional anisotropies $[33,34]$. Therefore, at higher volume concentration, the antenna resonance equation is no longer applicable [35]. When the wires are overlapped with one another as shown in the optical images of the dispersed wires (Fig. 2), the wires form clusters and the resonance frequency will be brought down to lower frequency [36]. The overlapping could be also responsible for the additional resonances seen for the composites containing the $30 \mathrm{~mA}$ annealed wires B [35]. Finally, the loading effect of the Pyrex glass coating should also be taken into account. The typical permittivity of Pyrex glass (4-10) is larger than that of silicone $(\sim 2)$, the resonance frequency is consequently shifted down [36].
In order to exploit the concept of plainification in programming wave scattering of microwire composites, we built several vertical-interface composites with varied compositions. With respect to the scattering properties of composites containing a combination of equal loading of as-cast A and annealed wires X (Fig. 5, right panel), two transmission windows are observed together with dips in reflection and absorption spectra (Fig. 5d-f). The dispersion of wires in vertical-interface composites is also confirmed by manufacturing several samples and the transmission trends are intimately closer to each other as shown in Figure S1b. The wires behave as a transmitting structure with band-pass feature in that condition. The transmission windows might originate from the coexistence of the separated regions in the composite. Due to the differences in conductivity and permittivity between the 


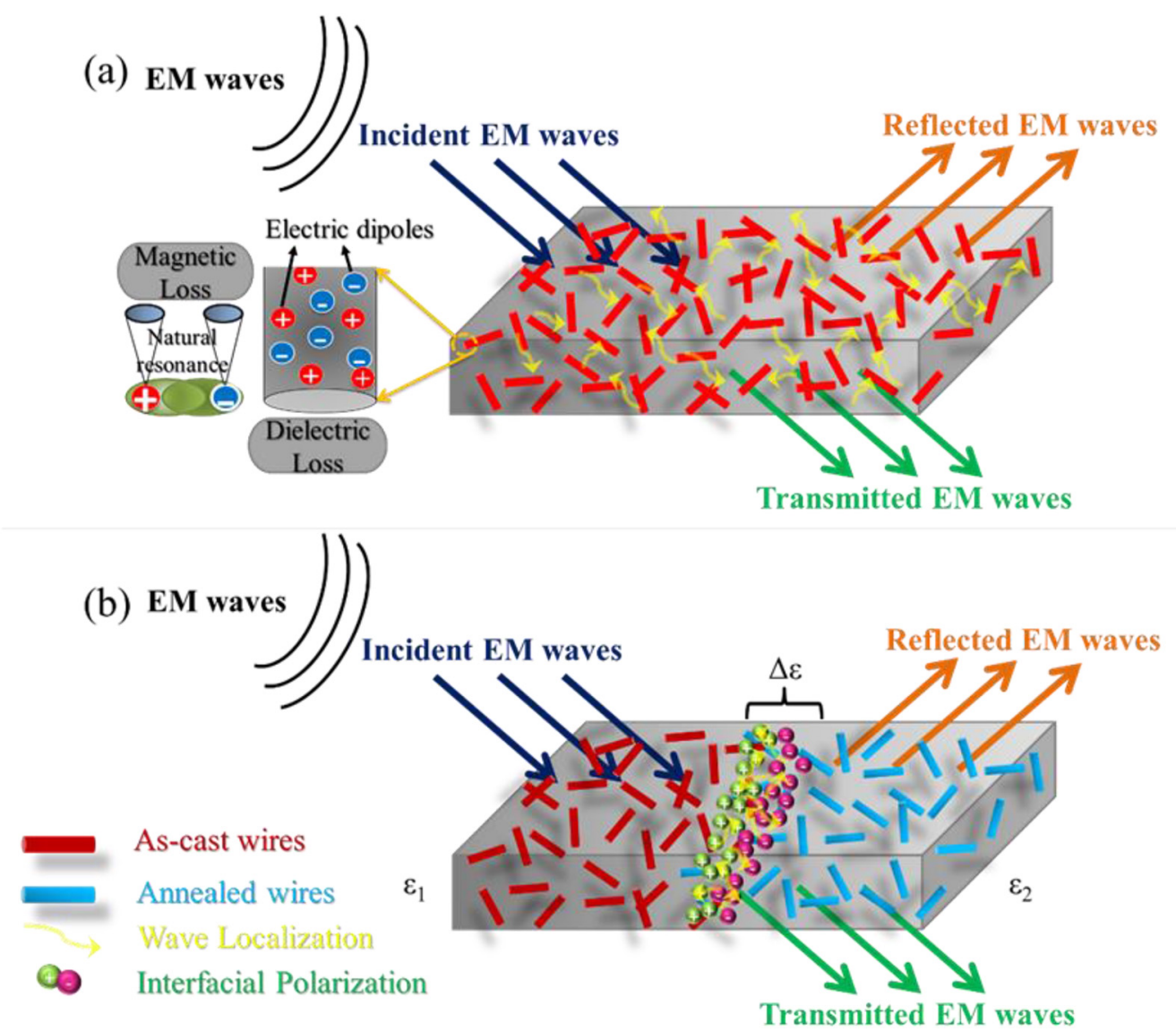

Fig. 7. Proposed scattering mechanisms in the randomly dispersed short-cut microwire composites: (a) composites containing the same type of wires; (b) composites containing as-cast wires and annealed wires in separated regions.

left and right regions of the sample, the vertical interface becomes the center of charge accumulation under the electric field. These space charges modify the field distribution and lead to an additional contributing to the overall dielectric properties of the composite and hence the electromagnetic response. Moreover, the transmission spectra move almost as a single unit to higher frequencies with lower magnitude as the $30 \mathrm{~mA}$ annealed wires (B) are replaced by the $40 \mathrm{~mA}$ annealed wires (C). Such changes are related to the gradual relief of internal stresses and induced structural relaxation experienced during the annealing process.

We move forward to tune the scattering properties of the composites by moving one region of the sample relative to another through variation in wire volume fraction (Fig. 1b). Incorporating majority of as-cast wires with respect to annealed wires ( 33 wires to 17 wires, respectively), results in distorted broad passbands and associated reflection and absorption peaks across the whole frequency range (Fig. 6, right panel). In this case, the electromagnetic response is influenced mainly by the as-cast wires that contribute to the appearance of the passbands and by the interface between the two regions, which results in the splitting of the transmission spectra. When more annealed wires than as-cast wires (17 wires to 33 wires, respectively) are present, the composites behave as a stop band structure showing the dominant contribution of annealed wires to the electromagnetic signature. Similar to the case of composites containing only annealed wires B (Fig. 5a), dual stopbands are noticed in the $\mathrm{A}(17)+\mathrm{B}(33)$ composites but with broader bandwidth (Fig. 6d). The single stopband or transmission dip found in composites containing only annealed wires $\mathrm{C}$ (Fig. 5a) is also displayed in the A $(17)+\mathrm{C}(33)$ composites, but a much deeper transmission minimum is present. It follows that the tunable band features can be modulated by varying the vertical interfacial region of the composites.

Now let us discuss the mechanisms behind the scattering response of the studied short-cut randomly dispersed composites. Note that all the composites under discussion are non-percolating due to the existence of glass coating and thus, there is no concern that the formation of a conductive network will hinder their interactions with the microwave [37]. The electromagnetic wave is scattered by inhomogeneities in the medium, and when such inhomogeneities are randomly distributed, the wave undergoes a large number of scattering events at random positions (Fig. 7a).

Different regimes of scattering are noted depending on the degree of randomness, ranging from multiple scattering to strong wave localization in which the waves tend to be "localized" or "trapped" in the random media leading to 
reduced transmission $[38,39]$. The attenuation of the incident wave by the microwires occurs through loss phenomena originated from dielectric and magnetic losses, which can be controlled by changing the surface impedance and magnetic anisotropy in the wires [28,40]. From Kittel's equation [41], the ferromagnetic resonance frequency $f_{r m}$ is expressed as:

$$
f_{r . m}=\frac{g}{2 \pi} \sqrt{\left(H_{a}+4 \pi M\right)\left(H_{a}+H\right)}
$$

where $g$ is the gyromagnetic ratio, $H_{a}$ is the magneto-elastic anisotropy field which depends on the wire's dimensions, $M$ is the magnetization and $H$ is the applied external magnetic field. Therefore, apart from the length of the wires, the resonance frequency is also controlled by changes in internal anisotropy of the wires as per equations (2) and (3). In the present case, such quantities were modified through current annealing as demonstrated by the hysteresis loops and impedance response (Sect. 3.1).

There are in general a number of critical parameters that control the microwave response of the studied composites, such as intrinsic properties of the wires, wire concentration and the in-built interface between the two different regions. For composites incorporating the same type of wires (Fig. 7a), the main factor influencing the scattering corresponds to the structure of the wires after the annealing process. As current annealing lowered the wire surface impedance (Fig. 4b), the scattering at the resonance increased and the rate of transmission was low [28]. However, as mentioned before, due to the closeness of crystallization and slightly larger anisotropy for the $40 \mathrm{~mA}$ annealed wires, the surface impedance increased leading to higher transmission (Fig. 5a). In the case of composites including the separated regions, apart from the structure of the wires, the role of the introduced vertical interface and wire concentration on wave propagation should also be considered. Electromagnetic waves are generally strongly localized near the interface of the two different media (Fig. 7b). When the incident wave hits the interface between the two different media with unequal dielectric and magnetic properties, the amount of wave transmitted or reflected varies with the polarization state at the vertical interface. Each composite sample is labelled from 1 to 6 , representing different states of polarization between the two regions across the interface $(\Delta \varepsilon)$ triggered by wire composition and filler microstructure variations (Fig. S2). Various dielectric dispersion behaviors are shown in the permittivity plots, ranging from almost constant response (samples 1 and 3) to relaxation (samples 2, 4 and 5) and resonance type (sample 6 ). It is revealed that, in addition to wire conductivity and impedance [28,42,43], the introduced vertical interface plays a major role in formulating the electromagnetic patterns. It follows that, through controlling the composition in each region, the discrepancy between their permittivity (the ability to restrict and accumulate charges) is varied, giving rise to disparate interactions between the composites and electromagnetic waves. It should also be noted that, fluctuations in wire loading increase the apparent attenuation due to scattering because of increased impedance contrasts [44,45].
Our approach demonstrates new opportunities for developing advanced microwire metacomposites by combining both strategies: microstructure modulation and plainification. Tunable properties in traditional metacomposites are achieved here by a simplified structure, constant filler content and tailored interfaces at a different length scale. For instance, unlike magnetic field-tuned scattering behavior achieved in previous studies [46-48], tunable microwave band-stop and band-pass behaviors are realized exempted from external magnetic fields. This eases the application of the studied composites in compact devices or light-weight structures where extra magnets are considered a burden. It also shows the potential of extrapolating the material plainification concept initially proposed in metallic alloys to composite media in realizing tunable performance without compromising lightweight design and structural integrity. It is worth pointing out that the passband and stopband performance with desired bandwidths or tunable negative electromagnetic properties could be further achieved by increasing fillers-concentration, hybridization with other functional fillers, inclusions alignment and supporting dielectric substrate modification. Relevant work is underway.

\section{Conclusions}

We have successfully realized tunable microwave passband and stopband features in randomly dispersed microwire metacomposites through wire microstructure and vertical interfacial region modulation based on a plainification design philosophy. Structural relaxation of the current annealed wires reflected in the change of magnetic, impedance and electrical properties which in turn influenced the scattering properties of the wire composites. For the composites incorporating the same type of wires, a marked transition from band-pass to band-stop behavior was shown when replacing as-cast wires by annealed wires due to refinement of circumferential anisotropy and lower impedance after annealing treatment. When a vertical interface was formed from incorporating wires of different structures in separated regions, double transmission windows with different amplitudes were obtained. The differences in conductivity and permittivity of charge carriers across the interface resulted in an interface-induced polarization which affected the wave propagation characteristics in the composites. Such vertical interface was modified by altering one of the regions through variations in wire volume concentration, enabling further tunable scattering spectra from broad passbands to stopbands with variable intensity. The advantages of large microwave tunability with low and constant filler loading and a relatively simple structure make the proposed composites attractive for the design of band-stop and band-pass filters.

\section{Supplementary Material}

Supporting information provided by the authors.

The Supplementary Material is available at https://www. epjam.edp-open.org/10.1051/epjam/2021003/olm. 
This work was supported by ZJNSF No. LR20E010001 and No. LY20E010005, Peng is indebted to the support by the Fundamental Research Funds for the Central Universities.

\section{References}

1. F.X. Qin, H.-X. Peng, V.V. Popov, L.V. Panina, M. Ipatov, V. Zhukova, A. Zhukov, J. Gonzalez, J. Appl. Phys. 109, 07A310 (2011)

2. L.V. Panina, M. Ipatov, V. Zhukova, J. Estevez, A. Zhukov, Mater. Res. Soc. Symp. Proc. 1312, 313 (2011)

3. F.X. Qin, H.-X. Peng, N. Pankratov, M.H. Phan, L.V. Panina, M. Ipatov, V. Zhukova, A. Zhukov, J. Gonzalez, J. Appl. Phys. 108, 044510 (2010)

4. Y. Luo, H.-X. Peng, F.X. Qin, M. Ipatov, V. Zhukova, A. Zhukov, J. Gonzalez, Appl. Phys. Lett. 103, 251902 (2013)

5. F.X. Qin, H.-X. Peng, Prog. Mater. Sci. 58, 183 (2013)

6. M. Tejedor, B. Hernando, M.L. Sánchez, V.M. Prida, M. Vázquez, Sens. Actuat. A Phys. 81, 98 (2000)

7. D.P. Makhnovskiy, L.V. Panina, J. Appl. Phys. 93, 4120 (2003)

8. G. Ababei, C.S. Olariu, N. Lupu, H. Chiriac, J. Appl. Phys. 117, 17A502 (2015)

9. A. Uddin, F.X. Qin, D. Estevez, S.D. Jiang, L.V. Panina, H.-X. Peng, Compos. Part B Eng. 176, 107190 (2019)

10. A. Uddin, D. Estevez, F.X. Qin, H.-X. Peng, J. Phys. D Appl. Phys. 53, 155302 (2020)

11. Y. Li, F.X. Qin, L. Quan, H. Wei, Y. Luo, H. Wang, H.-X. Peng, Carbon 153, 447 (2019)

12. M. Zhang, Z. Jiang, X. Lv, X. Zhang, Y. Zhang, J. Zhang, L. Zhang, C. Gong, J. Phys. D. Appl. Phys. 53, 02LT01 (2020)

13. M. Zhang, X. Fang, Y. Zhang, J. Guo, C. Gong, D. Estevez, F.X. Qin, J. Zhang, Nanotechnology 31, 275707 (2020)

14. X. Li, K. Lu, Science 364, 733 (2019)

15. H. Zhao, Y. Ren, B. Yang, G. Qin, J. Alloys Compd. 811, $152002(2019)$

16. P. Xie, W. Sun, Y. Liu, A. Du, Z. Zhang, G. Wu, R. Fan, Carbon 129, 598 (2018)

17. P. Xie, K. Sun, Z. Wang, Y. Liu, R. Fan, Z. Zhang, G. Schumacher, J. Alloys Compd. 725, 1259 (2017)

18. Z.C. Shi, R.H. Fan, Z.D. Zhang, L. Qian, M. Gao, M. Zhang, L.T. Zheng, X.H. Zhang, L.W. Yin, Adv. Mater. 24, 2349 (2012)

19. P. Xie, Z. Zhang, Z. Wang, K. Sun, R. Fan, Research 2019, 1021368 (2019)

20. V.S. Larin, A.V. Torcunov, A. Zhukov, M. Vazquez, L.V. Panina, J. Magn. Magn. Mater. 249, 39 (2002)

21. H. Chiriac, T.A. Óvári, Prog. Mater. Sci. 40, 333 (1996)

22. L.V. Panina, F.X. Qin, V. Zhukova, H.-X. Peng, J. Tang, A. Zhukov, M. Ipatov, J. Gonzalez, M.H. Phan, J. Non-Cryst. Solids 355, 1380 (2009)
23. P. Klein, R. Varga, J. Onufer, J. Ziman, G.A. BadiniConfalonieri, M. Vazquez, Acta Phys. Pol. A 131, 681 (2017)

24. J. Eckert, N. Mattern, W.T. Kim, D.H. Kim, E.S. Park, Prog. Mater. Sci. 58, 1103 (2013)

25. R.B. Marks, J.A. Jargon, J.R. Juroshek, 48th ARFTG Conf. Dig. 30, 38 (1996)

26. M.H. Phan, H.X. Peng, M.R. Wisnom, S.C. Yu, N. Chau, Compos. Part A Appl. Sci. Manuf. 37, 191 (2006)

27. A. Uddin, S.A. Evstigneeva, A. Dzhumazoda, M.M. Salem, M.G. Nematov, A.M. Adam, L.V. Panina, A.T. Morchenko, J. Phys.: Conf. Ser. 917, 082011 (2017)

28. D.P. Makhnovskiy, L.V. Panina, C. Garcia, A.P. Zhukov, J. Gonzalez, Phys. Rev. B 74, 064205 (2006)

29. N.A. Buznikov, J. Phys. D Appl. Phys. 43, 055002 (2010)

30. M.G. Nematov, A.M. Adam, L.V. Panina, N.A. Yudanov, A. Dzhumazoda, A.T. Morchenko, D.P. Makhnovskiy, F.X. Qin, J. Magn. Magn. Mater. 474, 296 (2019)

31. Z. Wang, L. Liu, L. Li, X. Li, F. Zu, Phase Transit. 86, 396 (2013)

32. E.M. Vinod, K. Ramesh, K.S. Sangunni, Sci. Rep. 5, 8050 (2015)

33. L. Sampaio, P. Vargas, D. Altbir, E.H.C. Sinnecker, M. Knobel, J. Magn. Magn. Mater. 249, 60 (2002)

34. D. Yong-Jiang, J. Jian-Jun, D. Gang, T. Bin, B. Shao-wei, H. Hua-hui, Trans. Nonferrous Met. Soc. China 17, 1352 (2007)

35. L. Liu, S.M. Matitsine, Y.B. Gan, K.N. Rozanov, J. Appl. Phys. 98, 063512 (2005)

36. L. Liu, Z.H. Yang, L.B. Kong, P. Li, C.H. Poo, Asia-Pacific Symp. Electromagn. Compat. APEMC 2012 - Proc. 12, 633 (2012)

37. H.-X. Peng, F.X. Qin, M.H. Phan, Ferromagnetic Microwire Composites: From Sensors to Microwave Applications (Springer International Publishing, Switzerland, 2016), pp. 1-245

38. J. Luo, Z.H. Hang, C.T. Chan, Y. Lai, Laser Photonics Rev. $\mathbf{9}, 523(2015)$

39. M.I. Mishchenko, Rev. Geophys. 46, RG2003 (2008)

40. Y.L. Xu, A. Uddin, D. Estevez, Y. Luo, H.-X. Peng, F.X. Qin, Compos. Sci. Technol. 189, 108022 (2020)

41. V. David, G. Ababei, H. Chiriac, I. Nica, V. Dafinescu, Rev. Sci. Instrum. 83, 014701 (2012)

42. A. Lagarkov, A. Sarychev, Phys. Rev. B 53, 6318 (1996)

43. D.P. Makhnovskiy, L.V. Panina, D.J. Mapps, A.K. Sarychev, Phys. Rev. B 64, 134205 (2002)

44. A.Z. Genack, N. Garcia, J. Opt. Soc. Am. 10, 125 (1993)

45. M. Van Der Baan, Geophys. J. Int. 145, 631 (2001)

46. L.V. Panina, M. Ipatov, V. Zhukova, J. Gonzalez, A. Zhukov, Intech Open 2, 432 (2015)

47. Y. Luo, D. Estevez, F. Scarpa, L.V. Panina, H. Wang, F.X. Qin, H.-X. Peng, Research 2019, 3239879 (2019)

48. Y. Luo, F.X. Qin, F. Scarpa, J. Carbonell, M. Ipatov, V. Zhukova, A. Zhukov, J. Gonzalez, L.V. Panina, H.-X. Peng, J. Magn. Magn. Mater. 416, 299 (2016)

Cite this article as: Azim Uddin, Faxiang Qin, Diana Estevez, Hua-Xin Peng, Vertical interface augmented tunability of scattering spectra in ferromagnetic microwire/silicone rubber metacomposites, EPJ Appl. Metamat. 8, 10 (2021) 